\title{
Interactive Spatial Analytics for Human-Aware Building Design
}

\author{
Muhammad Usman \\ York University \\ Toronto, Canada \\ usman@eecs.yorku.ca \\ Glen Berseth \\ University of British Columbia \\ Vancouver, Canada \\ gberseth@cs.ubc.ca
}

\author{
Davide Schaumann \\ Rutgers University \\ Piscataway, USA \\ davide.schaumann@gmail.com \\ Mubbasir Kapadia \\ Rutgers University \\ Piscataway, USA \\ mk1353@scarletmail.rutgers.edu
}

\author{
Brandon Haworth \\ York University \\ Toronto, Canada \\ brandon@eecs.yorku.ca \\ Petros Faloutsos \\ York University, UHN-TRI \\ Toronto, Canada \\ pfal@eecs.yorku.ca
}

\begin{abstract}
We present a computational spatial analytics tool for designing environments that better support human-related factors. Our system performs both static and dynamic analyses: the first relates to the building geometry and organization, while the second additionally considers the crowd movement in the space. The results are presented to the designers in the form of numerical values, traces and heat maps displayed on top of the floor plan. We demonstrate our approach with a user study whereby novice architects have tested the proposed approach to iteratively improve a building accessibility in real-time with respect to a selected number of static and dynamic metrics. The results indicate that the users were able to successfully improve their design solutions and thus generate more human-aware environments. The usability and effectiveness of the tool where also measured, yielding positive scores. The modular and flexible nature of the tool enables further extension to incorporate additional static and dynamic spatial metrics.
\end{abstract}

\section{CCS CONCEPTS}

- Computing methodologies $\rightarrow$ Visual analytics; Modeling and simulation; $•$ Applied computing $\rightarrow$ Computer-aided design; Architecture (buildings); • Human-centered computing $\rightarrow E m$ pirical studies in visualization;

\section{KEYWORDS}

Spatial analytics, human-aware design, architecture, crowd simulation, space analysis, navigation

\section{ACM Reference Format:}

Muhammad Usman, Davide Schaumann, Brandon Haworth, Glen Berseth, Mubbasir Kapadia, and Petros Faloutsos. 2018. Interactive Spatial Analytics for Human-Aware Building Design. In MIG '18: Motion, Interaction and Games (MIG '18), November 8-10, 2018, Limassol, Cyprus. ACM, New York, NY, USA, 12 pages. https://doi.org/10.1145/3274247.3274503

Permission to make digital or hard copies of all or part of this work for personal or classroom use is granted without fee provided that copies are not made or distributed for profit or commercial advantage and that copies bear this notice and the full citation on the first page. Copyrights for components of this work owned by others than the author(s) must be honored. Abstracting with credit is permitted. To copy otherwise, or republish, to post on servers or to redistribute to lists, requires prior specific permission and/or a fee. Request permissions from permissions@acm.org.

MIG '18, November 8-10, 2018, Limassol, Cyprus

(c) 2018 Copyright held by the owner/author(s). Publication rights licensed to ACM. ACM ISBN 978-1-4503-6015-9/18/11...\$15.00

https://doi.org/10.1145/3274247.3274503

\section{INTRODUCTION}

Architectural design involves exploring a large set of solutions to identify the one(s) that better satisfy a wide set of performance criteria while abiding specific constraints [Kalay 2004]. This is an iterative process whereby design solutions are developed and then progressively refined to maximize the overall design performance [Rittel 1971]. In the last 60 years, computational tools have been developed to assist designers in such a process. They help them measure the performance of a proposed design solution mainly in terms of energy, light, structure and cost. However, one of the most important aspects of a building design - how buildings support the users' behaviors - is often left to an architect's knowledge, experience, and imagination, which can be partial or biased [Zeisel 1984]. As a result, design artifacts often do not perform as expected, leading to severe consequences in terms of the users' experience, productivity, and even safety [Lawson 2004].

Predicting and analyzing user-related factors in building designs, however, is a complex task. Traditional Computer-Aided Design (CAD) and Building Information Modeling (BIM) mostly represent geometric and semantic aspects of building components (e.g., walls, doors, slabs). In an effort to analyze human social and spatial behaviors in buildings, Hillier and Hanson [1984] developed Space-Syntax, a well-known configurational approach that uses graph-based spatial representations to infer users' behavior by measuring spatial relations and connectivity. This approach has been proven useful to test how alternative design options affect the movement of people as a function of visibility and spatial connectivity [Sailer et al. 2009]. Space-Syntax, however, ignores the dynamic aspects of human movement in space. A representation of such a kind cannot be simply inferred by spatial visibility and connectivity: it depends on users' attributes (e.g. velocity), their location in space at a given time, the distance from a target, as well as the movement of other people in the space. Dynamic crowd simulations' analyses are thus required to account for agents' movement in space in day-to-day and emergency life threatening situations [Chu et al. 2014; Yan and Kalay 2004].

To assist architects and designers in generating and analyzing human-aware design options, we propose an interactive spatial analysis tool that can quantify human-related quantitative aspects both using static geometric information as well as dynamic crowdrelated movement patterns. 


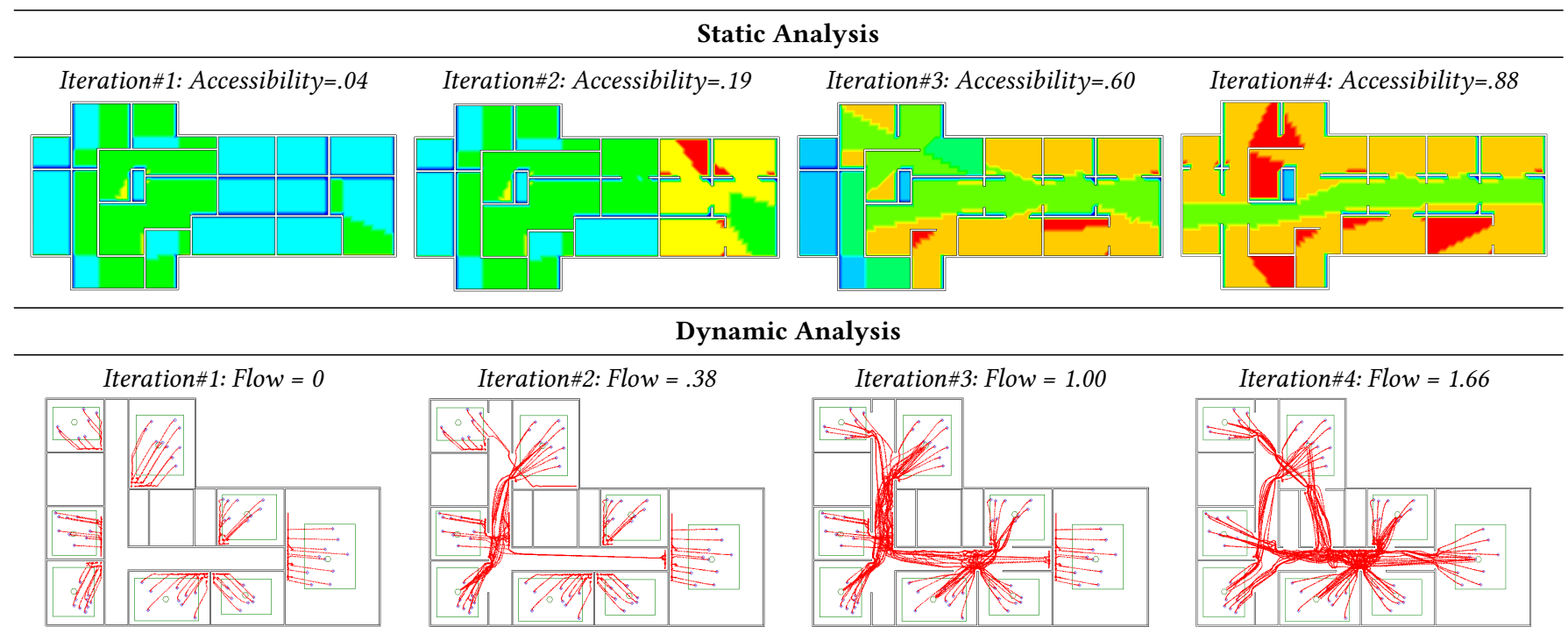

Figure 1: User designed environments using augmented Autodesk Revit ${ }^{\circledR}$ tools: $B$ - Revit-default with Static Analytics (TOP) and $C$ - Revit-default with Dynamic Analytics (BOTTOM). Spatial feedback allowed users to design more efficient environments in the succeeding iterations (informed decision-making). Both Accessibility and Crowd Flow values are highest in the last iteration. For static analysis, in the last iteration, heatmap showing fully connected environment with less accessible areas in the surroundings (ORANGE - RED) and high accessible areas at the center (GREEN).

Analyses of such a kind provide an intuitive way to identify problem areas, improve a building design, and compare design alternatives with respect to occupant-related factors by looking at real-time spatial and numerical visualizations. To demonstrate the proposed approach, we consider a representative set of quantitative measures from Space-Syntax and crowd simulations (details in Section 3). We hypothesize that, using this tool, building designers will be able to generate more human-aware building designs with respect to a selected number of metrics measured by the tool. We test our hypothesis with a user study. Novice users (people with moderate knowledge and expertise in architectural design and urban planning) were presented with a design task, and asked to increase the general accessibility of the designs by modifying architectural elements in the environment representing key portions of a building. The participants were asked to complete the design task using three different design methods. Method (A): using default Autodesk Revit tools, Method (B): using default Revit tools with assistance from spatial heatmap visualizations computed with Space-Syntax measures, and Method $(C)$ : using default Revit tools with assistance from spatial crowd-based visualizations (agents' traces) computed by running crowd simulations.

Our results indicate that the participants were able to design more accessible environments with the assistance from our tool. The participants also completed an evaluation study (USE Questionnaire) which asked them to rate the effectiveness and usability of each design method. The design method with feedback, Method (B): and Method $(C)$ : received high scores. The mean score of all USE dimensions were 80.90 and 80.92 respectively.

\section{RELATED WORK}

In architectural design, architects are required to evaluate multiple design solutions to identify the most satisficing one for a given spatial and social context [Simon 1969]. Due to the ill-structured nature of design problems, several competing design solutions may be generated, which affect the building performance in often unpredictable ways. Computational tools are thus needed to explore the implications of design decisions and make more informed decisions at the time a building is designed rather than after its construction and occupancy. Such tools are often coupled with existing CAD and BIM tools which provide only a geometric description of a set of building elements and their organization. Beyond geometry and spatial organization, such tools investigate the implications of a particular design in terms of energy efficiency [Clarke 2007], lighting [Rockcastle and Andersen 2014], daylight [Capeluto and Plotnikov 2017], structure [Weizmann et al. 2017], wind [Chen 2004], and construction efficency [Ben-Alon and Sacks 2017].

While many analyses of these kinds are currently embedded within (or connect to) well-established architectural design software like Rhinoceros or Autodesk Revit, analyses of how a building will affect its future inhabitants are mostly left to architects' knowledge and intuition. However, due to the complexity of built environments in terms of size, organization, multi-functionality, as well as types and number of occupants, predicting human behavior aspects can be challenging even for the most skilled architects. Spatial analysis tools have thus been proposed to assist architects in predicting and analyzing the mutual relations between a building layout and the presence and movement of its inhabitants. We categorize existing approaches into two major groups: static and dynamic spatial analyses. 


\subsection{Static Analysis of Human Behavior}

Static analysis represents human-related building aspects without the explicit modeling of people and time. These methods expand traditional CAD and BIM building models with information about the building's intended users and the activities they perform.

A well-known configurational approach named Space Syntax has been proposed by [Hillier and Hanson 1984] to analyze people movement in spaces using a graph-based representation (e.g. visibility graph) that measures spatial relations and connectivity. A wide set of analyses can thus be performed based on visibility graphs [Freeman 1978; Hölscher and Brösamle 2007; Turner et al. 2001]. Space-Syntax has been used to analyze human movement in various built environments [Gil et al. 2009; Hölscher et al. 2005; Stamps III 2002].

Other approaches provide static descriptions of people activities in space by coupling space models with user and activity models [Ekholm 2001; Maher et al. 1997]. Kim and Fischer [2014a,b] developed an automated method for mapping specific activities onto appropriate spaces. [Dzeng et al. 2015] describe a model to optimize functional use allocation based on a set of space-usage metrics including people movement and flow. Schultz and Bhatt [2012] and Bhatt et al. [2012] incorporated aspects of spatial cognition and reasoning into traditional space models to support designers' decision-making. Hölscher et al. [2009] study human navigation and way-finding in single and multi-level builidngs. Çekmiş et al. [2014] demonstrated a method to represent patterns of human inhabitation in open-planned spaces. Tashakkori et al. [2015] developed a space model that integrates indoor and outdoor information for emergency response facilitation. The work in Usman et al. [2017] investigates how people perceive spatial measures from Space-Syntax in different visual modes including blueprints, first-person and VR walkthroughs.

Overall, these methods provide a wide range of analyses without accounting for the dynamic movement and activities of building occupants.

\subsection{Dynamic Analysis of Human Behavior}

Dynamic analyses of human behavior aspects provide a time-based representation of a building in-use by their occupants. Beyond spatial information, these analyses require a specification of the users who will populate the space (e.g., a target destination, velocity, etc.), and the behaviors performed (e.g., moving to a target location, gathering with other agents, etc.). Different from the previous approach, dynamic analyses are considered as "simulations", and they are used when a specific phenomenon cannot be described using analytical methods (i.e. mathematical approaches) since the variables affecting the system interact in complex and unpredictable ways [Kalay 2004; Simon 1969].

Different models have been developed to represent people movement in virtual environments [Kapadia et al. 2015; Pelechano et al. 2016]. Particle-based approaches [Reynolds 1987] involve computing local-level interactions for speed, motion and relative position of each and every individual to deliver large crowd behavior. Some approaches have used social forces to model and show individual interactions [Helbing and Molnar 1995; Karamouzas et al. 2009].[Singh et al. 2011] presented a rule-based hybrid framework to avoid future collisions in crowd. [Van Den Berg et al. 2011] made use of reciprocal velocity obstacles so that velocity space can be computed for collision avoidance. An affordance based framework is proposed to compute space-time plan [Kapadia et al. 2009]. Event-driven approaches coordinate the behavior of large groups of agents to perform coordinated behaviors [Kapadia et al. 2016; Schaumann et al. 2017b]. More recently, researchers have started using data-driven techniques that adopt deep learning and reinforcement learning techniques to capture more realistic crowd behaviors [Heess et al. 2017; Peng et al. 2017]. Haworth et al. [2017b] presented a tool to study and evaluate the effects of pillar and obstacle placements on pedestrian flows during evacuation scenarios. However, it only supports trivial environment structures and doesn't allow to analyze full-scale buildings. Also, it is focused to analyze crowd interaction with pillars and obstacles only and doesn't take into account overall layout structure of a building. Chakraborty et al. [2017] presented a tool that allows the co-designing of the floor plans through simulation guided gamification approach. The designer or an architect set certain constraints for the floor plan and then the users co-design the floor plan while fulfilling those constraints. However, it's a very trivial system and doesn't take into account full-scale real environments and the kind of constraints associated with those environments. Wohl et al. [2016] also presented a gamification approach to model floor plans (game levels) by crowd-sourcing for fire evacuation scenarios through simulated crowds.

Crowd simulation techniques are more powerful than static techniques since they account for dynamic changes of the environment as well as the position of its inhabitants. However, they can be computationally expensive depending on the scale and complexity of the environment and characteristics of the simulation technique being used.

\subsection{Using Human Behavior Analysis Tools in Architectural Design}

Several approaches have been proposed to represent specific aspects of human behavior in architectural design using a combination of static and dynamic analyses. Such approaches include pedestrian movement [Yan and Kalay 2004], emergency egress [Chu et al. 2014; Pan et al. 2007], occupant presence and actions to support energy analyses [Goldstein et al. 2010], people movement in university buildings [Shen et al. 2013], and collaborative medical procedures in hospitals [Schaumann et al. 2017a]. Nonetheless, how static and dynamic human-related analysis tools are actually used in architectural design to support architects' decision-making is still a relatively under-studied topic.

Preliminary studies by Hong et al. [2016] and Hong and Lee [2018] indicated that human behavior analyses can support iterative refinement of design solutions in terms of day-to-day and emergency behaviors. Such studies, however, measured only few iterations over the course of an academic semester. We argue that in order to favor the use of static and dynamic spatial analyses, more advanced methods must be developed that compute design analyses in a fast-paced fashion, provide meaningful visual and numerical results that can be easily interpreted, are intuitive to use, and are connected with existing CAD or BIM tools. 


\subsection{Our Contribution}

In this work we demonstrate how architects and building designers can make use of quantitative and visual feedbacks using static and dynamic analysis to analyze their designs in real-time, not just at the very end, but at each phase during the design process to make intelligent and timely informed decisions. For demonstration purposes, we integrate our system into professional industry pipeline (e.g. Autodesk Revit ${ }^{\circledR}$ ), and we consider a representative number of metrics for both static and dynamic analyses. A user study evaluates the effectiveness of spatial analytics and visualizations in generating more human-aware design solutions.

\section{SPATIAL ANALYTICS FOR BUILDING DESIGN}

The proposed tool integrates into an architectural design workflow both static and dynamic spatial analyses to support architects' decision-making.

\subsection{Static Analysis of Building Designs}

As static analysis, we use Space-Syntax processes [Bafna 2003; Hillier and Hanson 1984]. A Visibility Graph [Turner 2001] is used to decompose an architectural layout into a graph to analyze the inter-visibility relations within architectural spaces. To construct a visibility graph, we sample a space using a homogeneous grid. All the points (cells) in the grid are the vertices $\left(V_{V G}\right)$ of the visibility graph. After that, we compute the lines of sight between the grid cells. If two grid cells are visible to each other (i.e. they have an unobstructed sight of view between them), there exists an edge $\left(E_{V G}\right)$ between corresponding vertices in the graph. Once the visibility graph is constructed, we can compute a selected number of Space-Syntax metrics that measure salient space characteristics. These are Accessibility, Visibility and Organization of Space.

3.1.1 Accessibility. It relates to the minimum average distance from a point to any other point in space. In other words, accessibility measures the struggle and difficulty of navigating the space from a given standpoint to other areas in space. In terms of graph, a vertex with high accessibility is connected to other vertices of the visibility graph through a smaller sequence of vertices.

Accessibility is measured as negative Tree Depth. Let all the graph trees whose root is $v_{i}$ in $V G$ forming a forest $F_{i}$, then rank of a tree $T_{i}$ with minimum depth in the forest $F_{i}$ is the Tree Depth $\left(\right.$ Dep $\left._{i}\right)$.

$$
\operatorname{Dep}_{i}=\operatorname{rank}\left(T_{i}\right)
$$

3.1.2 Visibility. It is the unobstructed line of sight between vertices. High visible areas are more connected with the surrounding spaces and provide better field of view. Hence, they are better candidates to install security camera, door placements, safety signs, etc. Visibility can also relate to Openness of Space from a specific standpoint.

Visibility of a vertex $v_{i}$ refers to the Degree of Visibility of that vertex. In terms of graph, it is defined as the number of neighbours $\left(N_{i}\right)$ incident to that vertex, $v_{i} \in V_{V G}$, connected by the edges, $E_{i} \subset E_{V G}$.

$$
\operatorname{Deg}_{i}=\left|N_{i}\right|
$$

3.1.3 Organization. It relates to the navigational choices a person faces at a particular standpoint within a space. For example, how easily a person can plan and navigates through the environment or building space. Organization is measured in terms of Entropy. Navigating through areas with less entropy (i.e. less organized spaces) implies a higher chance for a person to get lost or confused.

Organization of a vertex $v_{i}$ relates to the Entropy $\left(E n t_{i}\right)$ at that vertex. It is predicted on a probability distribution $p_{i}(l v l)$ of a tree $T_{i}$ with $n_{i}^{l v l}$ vertices at each level, $l v l$.

$$
E_{i} t_{i}=-\Sigma \sum_{l v l=0}^{\operatorname{height}\left(T_{i}\right)} p_{i}(l v l) \log _{2} p_{i}(l v l)
$$

\subsection{Dynamic Analysis of Building Designs}

As dynamic analysis, we use crowd simulation techniques to model movements of virtual agents in the environments. We integrate three crowd models: Social Forces [Helbing and Molnar 1995], Reciprocal Velocity Obstacle [Van Den Berg et al. 2011] and a Rule-based Hybrid framework [Singh et al. 2011]. Note that this work doesn't compare the performance of these models with each other nor does it claims if one is better than other. The only focus is that dynamic crowd simulation techniques can be used to analyze the environments with respect to human-occupancy. Two salient crowd-related measures are considered, namely Crowd Flow and Travelled Distance. These measures have been widely used especially in the evacuation scenarios to analyze crowd dynamics w.r.t. environment layout [Berseth et al. 2015; Cassol et al. 2017; Haworth et al. 2017a,b]. A user sets the crowd configuration (e.g. crowd spawn positions, target locations (goals), simulation technique, number of frames to simulate, etc.) within Autodesk Revit ${ }^{\circledR}$ and runs a simulation. Once a simulation is completed, the crowd-related measures i.e. crowd trajectory, flow and travelled distance, are shown to the user as feedback from dynamic analysis.

3.2.1 Crowd Flow. Similar to vehicular traffic, pedestrian dynamics have also been studied in the context of environment traffic [Fruin 1971]. In this study, we define crowd flow as rate at which all the agents complete their final goals [Berseth et al. 2015; Haworth et al. 2017a].

Simulating a crowd $(C)$, where $A_{c} \subseteq A$ are the agents who completed the simulation and reach their final goals or targets $(G)$, within some conventional time threshold $\left(t_{\text {sim }}\right.$ : the maximum simulation time set be the user). Let the average completion time of all the agents in reaching their final goals is $t_{a}^{f}$. The overall crowd flow of a simulation becomes $f_{C}$ :

$$
\text { Flow }_{c}=\frac{\left|A_{c}\right|}{t_{a}^{f}}
$$

where $\left|A_{c}\right|$ indicates the cardinality of set $A_{c}$. Crowd flow is measured in agents/second.

3.2.2 Distance. Travelled distance relates to the path or trajectory followed by a virtual agent $(a \subseteq A)$ to reach its target $(G)$ in a given period of time $\left(t_{\text {sim }}\right)$. Travelled distance can be defined as:

$$
\text { Distance }_{i}=r_{a} t_{\text {sim }}
$$


where $r_{a}$ indicates the travel rate of agents and $t_{\text {sim }}$ is the given simulation time. For this study, we consider the average distance travelled by all the agents during the course of simulation. Distance is measured in meter $(m)$.

\subsection{Data Visualization}

The static spatial measures are computed for the given space configuration and these are quantitative data (i.e. numbers). We calculate them at each vertex in a visibility graph. To visualize them, we color-code their values as a heatmap and overlay it on top of the actual floor plan of the environment. Architects and building designers can then visualize these heatmaps to analyze and identify different kinds of interesting areas within given space. For example, to identify areas with high visibility so that emergency exit signs can be installed. For calculating an overall value of these measures over entire space, we take an average of the values of all the vertices in the visibility graph.

From crowd-based measures, crowd flow and distances are shown as numeric values since these numbers are easily understandable and people can infer to them from their daily-life experiences. Agents' traces (crowd trajectories) are also shown as qualitative data. It is a path that an agent in motion follows through space as a function of time. Architects and designers can visualize agents' trajectories to analyze and examine the interaction between environment and occupants.

\section{USER STUDY}

\subsection{Experiment Design}

The goal of this study is to evaluate the effectiveness and usability of the proposed spatial analytics and visualizations with respect to real world use in architectural design. The hypothesis is that the proposed tool provides better assistance compared to existing modeling software in generating increasingly more accessible design solutions with respect to human-occupancy.

Participants were invited to take part in a user study session. The study session is delivered in three parts. In all three parts, participants are asked to complete an architectural design task (more details in Section 4.4). Three different design methods are used, one for each part of the study. Design Method - A: uses the default tool i.e. standard Autodesk Revit ${ }^{\circledR}$ interface. Design Method - B: uses the augmented tool which exposes the static spatial analytics and visualizations (described in Section 3.1) along with the standard Autodesk Revit ${ }^{\circledR}$ interface. Design Method $-C$ : uses the augmented tool which exposes dynamic crowd-based analytics and visualizations (described in Section 3.2) along with the standard Autodesk Revit ${ }^{\circledR}$ interface. Each participant completed all three parts and used Design Method - A for the first part, Design Method - B for the second part and Design Method $-C$ for the third part of the study. Later in this paper, these design methods are also referred as $A$ or Method $-A, B$ or Method $-B$ and $C$ or Method $-C$ respectively. To prevent any sort of learning effect between participants, the experimental conditions are delivered with balanced Latin-square design for the selection of environments to complete the design tasks. For the augmented Revit ${ }^{\circledR}$ tools (Method $-B \& C$ ), participants are instructed to press the feedback button in order to update (refresh) the spatial visualizations to reflect the new design modifications.
The updating or refreshing of a spatial visualization is counted as one design iteration. Participants are allowed to commit as many design iterations as necessary within the duration time (as detailed in the following paragraph).

Before beginning the study session, each participant signed a consent form, was briefly instructed about the study design and spatial measures, completed two questionnaires to collect demographic information and domain knowledge and was given a demo (practice session) on how to use default (standard) and augmented Autodesk Revit ${ }^{\circledR}$ interfaces. Afterwards, the actual study session started. Participants were allowed 15 minutes to complete each part of a study. Participants were given another 10 minutes to complete both a domain-specific feedback questionnaire and a Usefulness, Satisfaction and Ease of use, USE [Lund 2001], questionnaire to evaluate the effectiveness and usability of spatial analytics and visualizations, after Method-B \& $C$. Overall, the whole study took 1 hour and 25 minutes of time at maximum.

All participants completed the user study on a Lenovo laptop with the following specifications: Intel(R) Core i7-6700HQ CPU @ 2.60GHz (8 CPUs), 12 GB of RAM (DDR4), Nvidia GeForce GTX 1060 (Graphics Card) and Microsoft Windows 10 Home (OS). The plugin itself is written in .NET programming language and is integrated into Autodesk Revit @ ${ }^{8}$ 2017. For running crowd simulations, SteerSuite [Singh et al. 2009], an open framework for crowd simulation and optimization techniques, is used.

4.1.1 Independent Variables. The three real-world environments as well as the apparatus (Design Method - A, B \& C) are considered independent variables.

4.1.2 Dependent Variables. Static spatial measures (1) Accessibility, (2) Visibility, and (3) Organization of the space, and dynamic crowd-based measures (4) Crowd Flow and (5) Distance, for users' modified environments were the primary dependent variables. The total number of design iterations and completion time in each part of the study session is also counted.

\subsection{Environments}

A variety of real-world environments including an Art Gallery, an Office and a Museum Space are used to illustrate the effectiveness of spatial analytics and visualizations. Figure 2 shows the layouts of these real-world environments. For the sake of this study, we removed all openings and doorways from the default layouts of these environments which were added by an actual architect firm. This is because we wanted users to modify the environments by adding openings, doorways and modifying other architectural elements, in order to make the space (e.g. rooms) connected (i.e. traversable from other spaces in the environment) because anyone of these spaces could be an office, meeting room, study or common area, cafeteria, or even a restroom, that someone needs to access.

As discussed in Section 3, static spatial measures are computed over entire space of an environment and then an average value for that environment is considered for the analytics. For crowdbased spatial analytics, 75 virtual agents are uniformly distribute in sub-groups and these sub-groups have diametric goals within environment to achieve maximum space coverage and to describe possible day-to-day use of the space (further details on design task is 


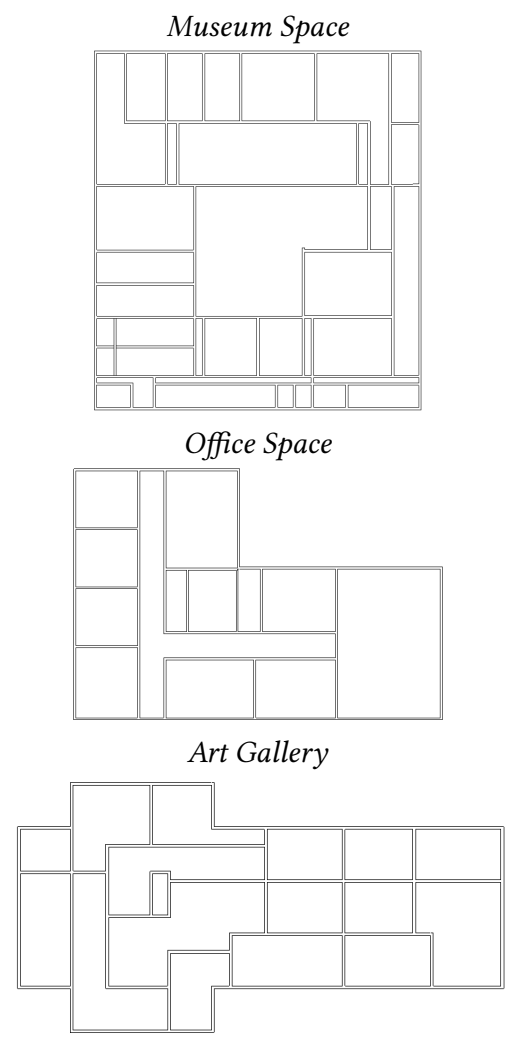

Figure 2: Three real-world environments are used in the study. We removed all the openings in the environments because we wanted study users to make these disconnected spaces connected by modifying the environments using proposed tools.

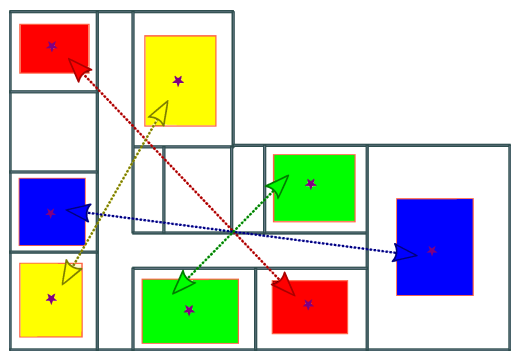

Figure 3: Initial crowd configuration showing spawn regions and desired goals or targets in a disconnected environment. Different from traditional evacuation scenarios, the crowd is uniformly distributed in sub-groups across the environment with diametric goals.

given in Section 4.4). Figure 3 shows an initial crowd configuration, spawn regions for agents' sub-groups and their diametric targets, in a disconnected environment for an Office Space.

\subsection{Participants}

15 people ( 8 female, 7 male and 1 non-binary/third-gender between 25 and 34 years of age) voluntarily participated in the user study. They were paid 10 dollars per hour as an appreciation for their time. The participants were mostly senior-level university students studying architecture and urban planning, and some even had technical and professional certifications in architecture designing.

For the sake of this study, we only recruited those participants who had above-average knowledge and experience in interpreting architectural floor plans, understanding of pedestrians' flow and Space-Syntax concepts, and had some hands-on experience of using architecture design tools e.g. Autodesk REVIT ${ }^{\circledR}$. Table 1 shows the demographic information collected from the recruited participants. It also shows the domain knowledge collected from the participants at the time of recruitment. Average scores for domain knowledge on a scale of 1 - 5 for ability to interpret and prior experience with architecture designs, prior experience in urban planning, and prior understanding of the considered static and dynamic spatial measures are 4.0, 4.0, 3.5, 3.9 and 3.5 respectively (self-reported).

\subsection{Experiment Task}

Participants are asked to add openings and pathways to a disconnected environment so people can traverse the environment in the most accessible way by modifying (but not entirely remove) architectural and geometrical elements like walls, pillars or other obstacles. Participants are not allowed to add more than two openings or doorways per room. Maximum time allowed to complete the design task is 15 minutes. Participants are notified 1 minute before the maximum allowed time to complete the task. If someone completes a task before-time, that participant can inform the experimenter to stop the design task.

At the end of a design task in Method - B \& $C$, participants are asked to complete two surveys: one to provide domain-specific feedback (see Table 2), and a Usefulness, Satisfaction and Ease of use, USE [Lund 2001] questionnaire to evaluate the effectiveness and usability of the spatial analytics, visualizations and the tool itself. Maximum time allowed to complete the surveys is 10 minutes. Participants are notified 1 minute before the maximum allowed time to complete the surveys.

\subsection{Results}

4.5.1 Task Completion Time and Elements'Modifications. On average, participants spend 5 minutes and committed 250 modifications of architectural elements (e.g. walls, pillars or other obstacles) in Method - A, 9 minutes and committed 130 modifications in Method - B and 8 minutes and committed 133 modifications in Method $-C$.

To compare users' design performances from augmented Revit ${ }^{\circledR}$ tools (Method - B \& $C$ ) with default tool (Method -A), we compute the same set of spatial measures in a post-study fashion for user modified designs from Method - A. This enables us to compare the design performances between Method - $A \& B$ for static spatial measures and between Method - $A \& C$ for dynamic crowd-based measures. 
Table 1: Demographic information and domain knowledge of user-study participants (self-provided).

Demographic Information

\begin{tabular}{|c|c|c|c|c|c|c|}
\hline \multicolumn{2}{|l|}{ Gender } & Sex & \multicolumn{2}{|l|}{ Age } & \multicolumn{2}{|c|}{ Country of Residence } \\
\hline Female: $8(53.3 \%)$ & \multirow{3}{*}{\multicolumn{2}{|c|}{$\begin{array}{l}\text { Female: } 8(53.3 \%) \\
\text { Male: } 6(40 \%) \\
\text { Intersex: } 1(6.7 \%)\end{array}$}} & \multirow{3}{*}{\multicolumn{2}{|c|}{25 - 34 years old: $15(100 \%)$}} & \multirow{3}{*}{\multicolumn{2}{|c|}{ Canada: $15(100 \%)$}} \\
\hline Male: $6(40 \%)$ & & & & & & \\
\hline Non-binary/Third-gender: $1(6.7 \%$ & & & & & & \\
\hline \multicolumn{7}{|c|}{ Domain Knowledge } \\
\hline & Poor & Below Average & Average & Above Average & Excellent & AVG. scale 1-5 \\
\hline $\begin{array}{l}\text { Ability to interpret architectural or } \\
\text { interior designs? }\end{array}$ & $0(0 \%)$ & $0(0 \%)$ & $0(0 \%)$ & $15(100 \%)$ & $0(0 \%)$ & 4.0 \\
\hline $\begin{array}{l}\text { Prior experience with architecture } \\
\text { or interior designs? }\end{array}$ & $0(0 \%)$ & $0(0 \%)$ & $0(0 \%)$ & $15(100 \%)$ & $0(0 \%)$ & 4.0 \\
\hline $\begin{array}{l}\text { Prior experience in urban planning } \\
\text { and design? }\end{array}$ & $0(0 \%)$ & $0(0 \%)$ & $8(53.3 \%)$ & $7(46.7 \%)$ & $0(0 \%)$ & 3.5 \\
\hline $\begin{array}{l}\text { Prior understanding of spatial } \\
\text { Space-Syntax measures? }\end{array}$ & $0(0 \%)$ & $0(0 \%)$ & $2(13.3 \%)$ & $13(86.7 \%)$ & $0(0 \%)$ & 3.9 \\
\hline $\begin{array}{l}\text { Prior understanding of pedestrian } \\
\text { movement (e.g. crowd flow)? }\end{array}$ & $0(0 \%)$ & $0(0 \%)$ & $7(46.7 \%)$ & $8(53.3 \%)$ & $0(0 \%)$ & 3.5 \\
\hline
\end{tabular}

Table 2: Opinion of participants on the effectiveness of spatial analytics and visualizations in real-life architecture designing and urban planning (self-reported).

Opinion of Participants on the Effectiveness of Spatial Visualizations in Real-Life Designing

\begin{tabular}{|c|c|c|c|c|c|c|}
\hline & Poor & Below Average & Average & Above Average & Excellent & AVG. scale 1-5 \\
\hline & \multicolumn{6}{|c|}{ Do you consider this tool as a valuable addition to traditional geometric modeling tools? } \\
\hline Method B & $0 \%$ & $0 \%$ & $0 \%$ & $53.3 \%$ & $46.7 \%$ & $4.46(89.2 \%)$ \\
\hline \multirow[t]{2}{*}{ Method C } & $0 \%$ & $0 \%$ & $0 \%$ & $40 \%$ & $60 \%$ & $4.60(92.0 \%)$ \\
\hline & \multicolumn{6}{|c|}{ In day-to-day work, do you think these analyses can help you make more informed decisions while designing } \\
\hline Method B & $0 \%$ & $0 \%$ & $0 \%$ & $86.7 \%$ & $13.3 \%$ & $4.13(82.6 \%)$ \\
\hline \multirow[t]{2}{*}{ Method C } & $0 \%$ & $0 \%$ & $0 \%$ & $93.3 \%$ & $6.7 \%$ & $4.06(81.2 \%)$ \\
\hline & \multicolumn{6}{|c|}{ Do you see this approach as helpful for improving architecture designs? } \\
\hline Method B & $0 \%$ & $0 \%$ & $6.7 \%$ & $93.3 \%$ & $0 \%$ & $3.93(78.6 \%)$ \\
\hline \multirow[t]{2}{*}{ Method C } & $0 \%$ & $0 \%$ & $6.7 \%$ & $93.3 \%$ & $0 \%$ & $3.93(78.6 \%)$ \\
\hline & \multicolumn{6}{|c|}{ To what extend do you believe these spatial metrics capture essential features of how people use spaces? } \\
\hline Method B & $0 \%$ & $0 \%$ & $26.7 \%$ & $73.3 \%$ & $0 \%$ & $3.73(74.6 \%)$ \\
\hline \multirow[t]{2}{*}{ Method C } & $0 \%$ & $0 \%$ & $6.7 \%$ & $86.7 \%$ & $6.7 \%$ & $4.00(80.0 \%)$ \\
\hline & \multicolumn{6}{|c|}{ Such visualization tools can be adopted into architectural design workflow pipeline? } \\
\hline Method B & $0 \%$ & $0 \%$ & $6.7 \%$ & $93.3 \%$ & $0 \%$ & $3.93(78.6 \%)$ \\
\hline Method C & $0 \%$ & $0 \%$ & $26.7 \%$ & $73.3 \%$ & $0 \%$ & $3.73(74.6 \%)$ \\
\hline
\end{tabular}

4.5.2 Static Spatial Analytics. Accessibility: a significant effect of Design Methods is found on participants' design performances at a significance level of $p<0.05$ for the three conditions $[\mathrm{F}(1,28)=$ $4.45=0.0439]$. A post-hoc comparison was then performed using the Tukey HSD test to compare across conditions. The test indicates that Method - B has higher effects than Method - A. Visibility: a significant effect of Design Methods is found on participants' design performances at a significance level of $p<0.05$ for the three conditions $[\mathrm{F}(1,28)=7.25=0.0118]$. A post-hoc comparison was then performed using the Tukey HSD test to compare across conditions. The test indicates that Method - $C$ has higher effects than Method A. Organization: no significant effect of Design Methods is found on participants' design performances at a significance level of $p<0.05$ 

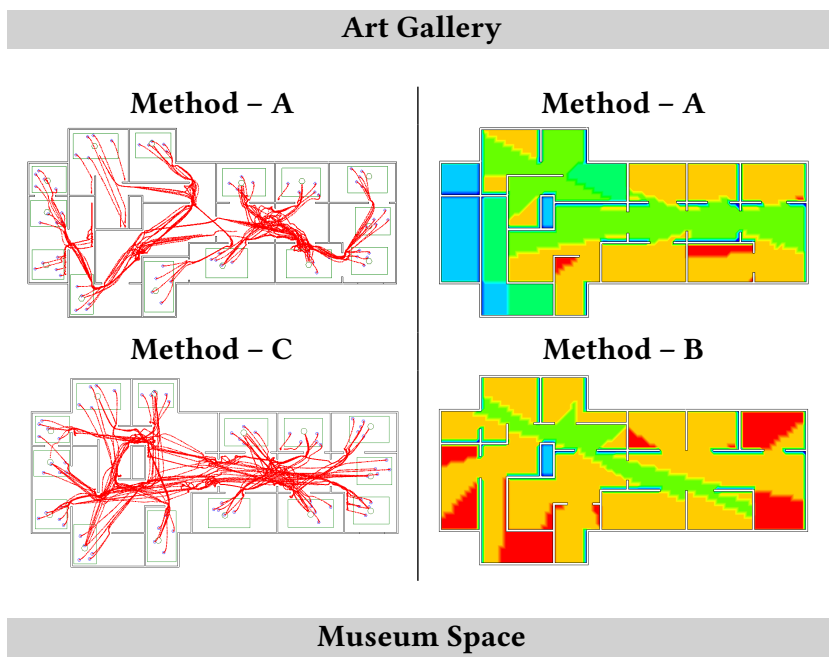

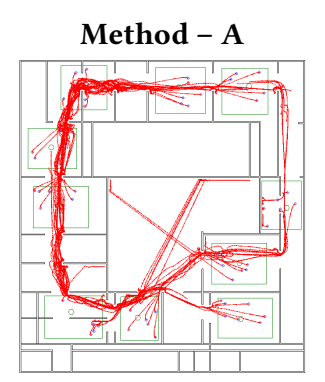

Method - C

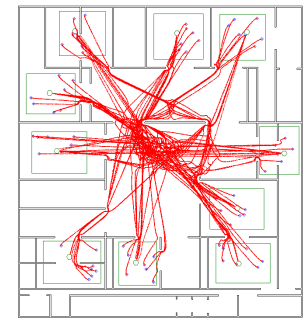

Method - A

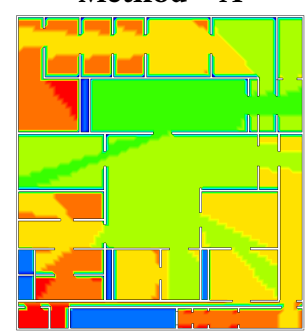

Method - B

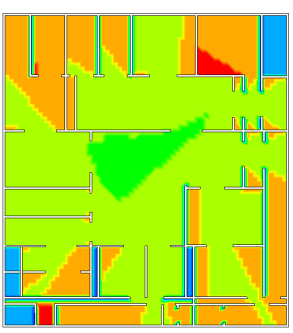

Figure 4: Comparing qualitative results for user modified designs between: Methods $-A \& B$ and $A \& C$. For augmented tools $(B \& C)$, designs from the final users' iterations are used. Users with augmented Revit ${ }^{\circledR}$ tools were able to design multi-route environments (more accessible).

for the three conditions $[\mathrm{F}(1,28)=0.04=0.8385]$. However, participants' performed better in Method $-B$ in general. Mean and Standard Deviation values are reported below.

Figure 5 shows box and whisker plots for static spatial measures for user designs from Design Method - A \& B. On the left are the results for Accessibilty, at the middle for Visibility and on the right for Organization of space. Since no spatial analytics and visualization feedback was available in Method - A, therefore, participants failed to perform well and their modified designs from standard Revit ${ }^{\circledR}$ tool produced comparatively low values for accessibility, visibility and organization of space. From Method - B, accessibility, visibility and organization values are reported for participants' best and final designs across all design task iterations. Art Gallery: Mean accessibility, visibility and organization values with standard deviation recorded from Method $-A$ are $0.70 \pm 0.19,0.10 \pm 0.06$ and $0.19 \pm 0.17$ respectively, whereas the values for final designs from Method $-B$ are $0.90 \pm 0.06,0.15 \pm 0.09$ and $0.23 \pm 0.09$ respectively. Office: Mean accessibility, visibility and organization values with standard deviation recorded from Method $-A$ are $0.81 \pm 0.18$, $0.65 \pm 0.31$ and $0.38 \pm 0.10$ respectively, whereas the values for final designs from Method $-B$ are $0.82 \pm 0.13,0.93 \pm 0.06$ and $0.25 \pm 0.18$ respectively. Museum: Mean accessibility, visibility and organization values with standard deviation recorded from Method $-A$ are $0.30 \pm 0.25,0.32 \pm 0.19$ and $0.63 \pm 0.20$ respectively, whereas the values for final designs from Method $-B$ are $0.40 \pm 0.25,0.69 \pm 0.13$ and $0.66 \pm 0.24$ respectively.

Table 3 shows the number of design iterations completed by the participants during the task in Design Method - $B$ and the corresponding Accessibility, Visibility and Organization values per iteration. Since all three environments were disconnected in the start, therefore, initial iterations have low accessibility, visibility and organization values. However, as the users completed more design iterations and made the environments accessible and connected, the accessibility, visibility and organization values increased. These values are color-coded from RED - GREEN, where RED highlights low values for these spatial measures and GREEN, high.

4.5.3 Dynamic Spatial Analytics. A significant effect of Design Methods is found on participants' design performances at a significance level of $p<0.05$ for the three conditions $[\mathrm{F}(1,28)=33.95=$ $2.9175 \mathrm{e}-06]$. A post-hoc comparison was then performed using the Tukey HSD test to compare across conditions. The test indicates that Method $-C$ has higher effects than Method $-A$.

Figure 6 shows box and whisker plots for dynamic crowd-based measures for user designs from Design Method - A \& $C$. On the left are the results for Crowd Flow and on the right for Distance travelled by the virtual agents during the course of simulation. From Design Method $-C$, crowd flow and travelled distances are reported for participants' best and final designs across all design task iterations. Art Gallery: Mean and standard deviation values for crowd flow and travelled distance recorded from Method $-A$ are $0.53 \pm 0.15$ and $41.42 \pm 2.52$ respectively, whereas the values recorded from Method $-C$ for the final designs are $1.01 \pm 0.24$ and $37.83 \pm 5.16$ respectively. Office: Mean and standard deviation values for crowd flow and travelled distance recorded from Method $-A$ are $0.94 \pm 0.07$ and $38.96 \pm 2.46$ respectively, whereas the values recorded from Method $-C$ for the final designs are $1.54 \pm 0.16$ and $38.08 \pm 4.74$ respectively. Museum: Mean and standard deviation values for crowd flow and travelled distance recorded from Method $-A$ are $0.50 \pm 0.35$ and $40.25 \pm 3.89$ respectively, whereas the values recorded from Method $-C$ for the final designs are $1.31 \pm 0.10$ and $34.45 \pm 6.69$ respectively. Crowd flow is measured in agents/second and travelled distance in meter.

Table 4 shows the number of design iterations completed by the participants during the task in Design Method $-C$ and the corresponding Crowd Flow values per iteration. Since all three environments were disconnected in the start, therefore, initial iterations have low crowd flows. However, crowd flow increased as the participants committed more design iterations and made the environment 
Table 3: Static spatial measures: Accessibility (LEFT), Visibility (MIDDLE) and Organization (RIGHT), from Space-Syntax, for the design iterations completed by the users in Design Method - B. Overall, users' design performance increased as they complete more iterations and have comparatively best results in their last iterations.

\begin{tabular}{|c|c|c|c|c|c|}
\hline \multirow{3}{*}{$\begin{array}{c}\text { Participant } \\
2\end{array}$} & \multicolumn{5}{|c|}{ Accessibility } \\
\hline & \multicolumn{5}{|c|}{ Art Gallery } \\
\hline & 0.02 & 0.51 & 1 & & \\
\hline 4 & 0.06 & 0.24 & 0.43 & 0.92 & \\
\hline 7 & 0.04 & 0.19 & 0.60 & 0.88 & \\
\hline 10 & 0.07 & 0.30 & 0.67 & & \\
\hline \multirow[t]{2}{*}{13} & 0.04 & 0.29 & 0.55 & 0.78 & \\
\hline & \multicolumn{5}{|c|}{ Office } \\
\hline 3 & 0.06 & 0.28 & 0.76 & 0.66 & 0.66 \\
\hline 6 & 0.03 & 0.16 & 1 & & \\
\hline 9 & 0.07 & 0.27 & 0.51 & 0.66 & \\
\hline 12 & 0.05 & 0.34 & 0.76 & & \\
\hline \multirow[t]{2}{*}{15} & 0.02 & 0.26 & 0.33 & 0.53 & \\
\hline & \multicolumn{4}{|c|}{ Museum } & \\
\hline 1 & 0.06 & 0.25 & 0.65 & 0.62 & \\
\hline 5 & 0.06 & 0.17 & 0.84 & & \\
\hline 8 & 0.04 & 0.13 & 0.23 & 0.53 & \\
\hline 11 & 0.05 & 0.33 & 0.59 & 0.61 & \\
\hline \multirow[t]{2}{*}{14} & 0.07 & 0.29 & 1 & & \\
\hline & 1 & 2 & 3 & 4 & 5 \\
\hline
\end{tabular}

\begin{tabular}{c|c|c|c|c}
\hline \multicolumn{5}{c}{ Visibility } \\
\hline \multicolumn{5}{c}{ Art Gallery } \\
0.06 & 0.17 & 0.34 & & \\
0.03 & 0.38 & 0.49 & 0.75 & \\
0.07 & 0.17 & 0.47 & 0.72 & \\
0.06 & 0.41 & 0.89 & & \\
0.06 & 0.36 & 0.66 & 1 & \\
\multicolumn{5}{c}{ Office } \\
0.07 & 0.31 & 0.64 & 0.89 & 0.84 \\
0.04 & 0.22 & 0.92 & & \\
0.08 & 0.31 & 0.52 & 0.63 & \\
0.04 & 0.40 & 0.93 & & \\
0.03 & 0.48 & 0.58 & 1 & \\
\multicolumn{5}{c}{ Museum } \\
0.06 & 0.19 & 0.54 & 0.55 & \\
0.05 & 0.30 & 0.74 & & \\
0.04 & 0.10 & 0.34 & 0.71 & \\
0.07 & 0.36 & 0.99 & 1 & \\
0.03 & 0.50 & 0.81 & & \\
$\mathbf{1}$ & $\mathbf{2}$ & $\mathbf{3}$ & $\mathbf{4}$ & $\mathbf{5}$ \\
\hline \multicolumn{5}{|c}{ Iterations } \\
\end{tabular}

\begin{tabular}{c|c|c|c|c}
\hline \multicolumn{5}{c}{ Organization } \\
\hline \multicolumn{5}{c}{ Art Gallery } \\
0.05 & 0.57 & 1 & & \\
0.06 & 0.36 & 0.57 & 0.98 & \\
0.05 & 0.27 & 0.66 & 0.92 & \\
0.07 & 0.40 & 0.77 & & \\
0.03 & 0.41 & 0.63 & 0.80 & \\
\multicolumn{5}{c}{ Office } \\
0.08 & 0.37 & 0.98 & 0.79 & 0.80 \\
0.06 & 0.18 & 0.98 & & \\
0.06 & 0.35 & 0.55 & 0.74 & \\
0.06 & 0.49 & 1 & & \\
0.05 & 0.34 & 0.43 & 0.66 & \\
\multicolumn{5}{|c}{ Museum } \\
0.08 & 0.42 & 0.90 & 0.90 & \\
0.05 & 0.29 & 1 & & \\
0.08 & 0.20 & 0.41 & 0.68 & \\
0.04 & 0.39 & 0.67 & 0.69 & \\
0.07 & 0.44 & 0.88 & & \multirow{2}{*}{$\mathbf{5}$} \\
\hline $\mathbf{1}$ & $\mathbf{2}$ & $\mathbf{3}$ & $\mathbf{4}$ & $\mathbf{5}$ \\
\hline \multicolumn{5}{|c}{ Iterations } \\
\end{tabular}

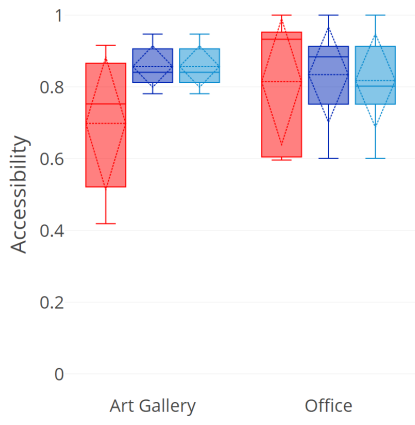

Accessibility
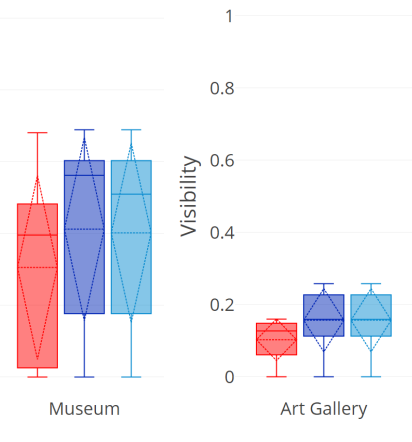

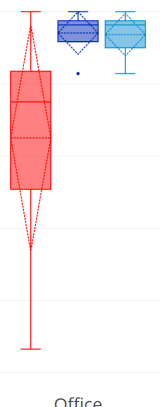

Office

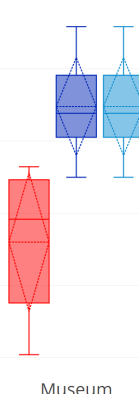

Museum

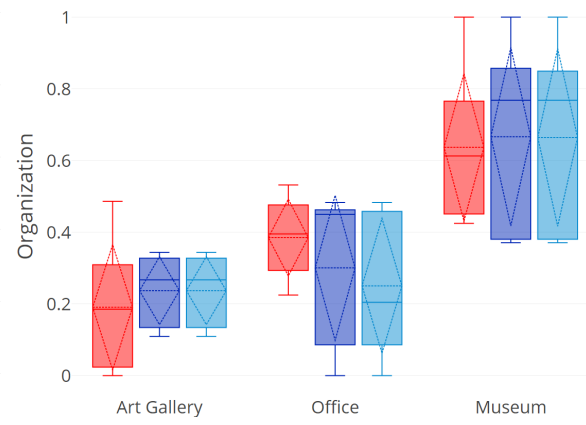

Organization

Figure 5: Showing results for static spatial measures Accessibility, Visibility and Organization from Space-Syntax for user modified environments from Method - A \& B. For Method - B, results from best and final design iterations are shown. Overall, user designs from the augmented Revit ${ }^{\circledR}$ tool $($ Method $-B)$ performed well and produced high values for accessibility, visibility and organization of space. More details in Section 4.5.2.

accessible and connected. Flow values are color-coded from RED GREEN, where RED highlights low crowd flow and GREEN, high.

A one-way between subjects analysis of variance was conducted. Statistical results indicate significant effect of Design Methods on participants' design performances. Furthermore, post-hoc tests using the Tukey HSD indicate that Method $-B$ \& $C$ have higher effects on design performances. Comparatively, user designs from Method - $B \& C$ achieved higher mean values for the static and dynamic spatial measures with low standard deviations which shows consistency among users with these design methods. Overall, as users completed more design iterations with augmented Revit ${ }^{\circledR}$ tools, they made accessible and more human-aware environments.
4.5.4 Qualitative Results. Figure 4 shows a selection of qualitative results for users' modified designs from standard (Method $-A$ ) and augmented (Method $-B \& C$ ) Revit ${ }^{\circledR}$ tools. Spatial visualizations indicate that with augmented tools, users made more informed decisions and successfully achieved multi-route spaces, making the environments more accessible. Figure 1 shows sample designs from the user study. Two set of environment designs are shown which were designed using augmented Revit ${ }^{\circledR}$ tools (Method $-B \& C$ ). Four design iterations are committed. Spatial feedback helped participants to design more efficient environments in the succeeding iterations. Both Accessibility and Crowd Flow values are 

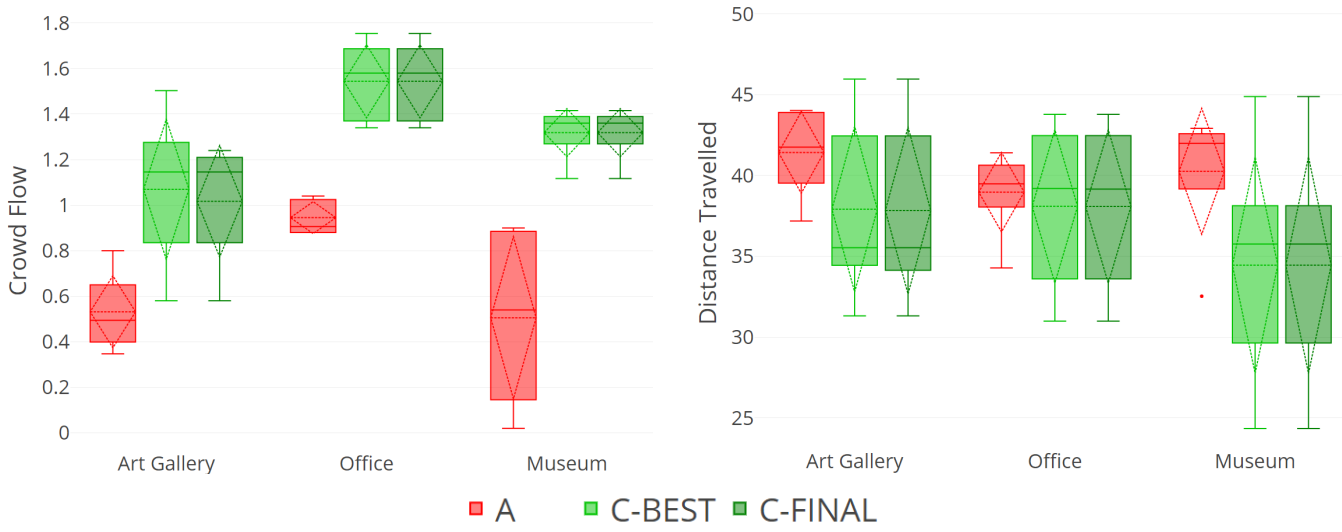

Crowd Flow

Distance Travelled

Figure 6: Showing results for Crowd Flow and Distance Travelled for user modified environments from Method - A \& C. For Method $-C$, results from best and final design iterations are shown. Overall, user designs from the augmented Revit ${ }^{\circledR}$ tool $($ Method $-C)$ performed well, produced high crowd flows and agents travelled less distances. More details in Section 4.5.3.

Table 4: The Crowd Flow values for design iterations completed by users in Method - C. Higher flows are considered good. Overall, the Crowd Flow increased as users completed more iterations and achieved the highest flow in last ineration.

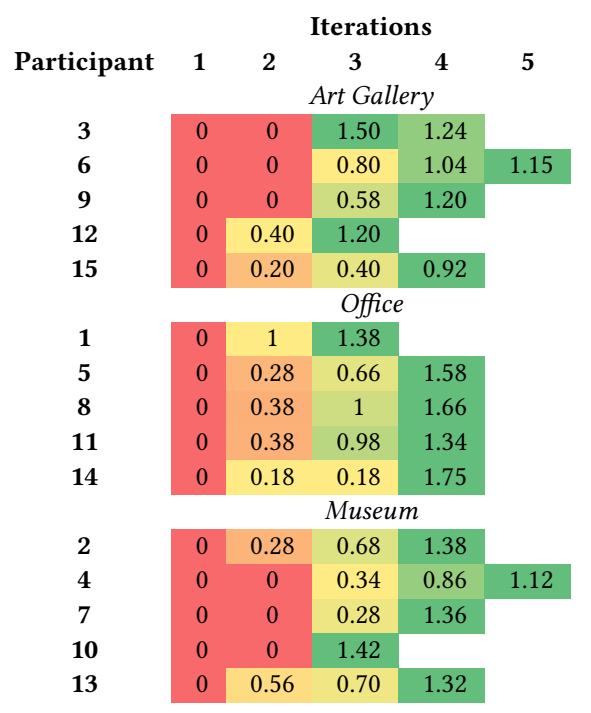

highest in the last iteration. Note that because of space limitations only Accessibility heatmaps are shown in the qualitative results.

4.5.5 Usability and Effectiveness of the Tool. To evaluate the effectiveness and usability of the augmented Revit ${ }^{\circledR}$ tools, participants completed a survey - Usefulness, Satisfaction and Ease of use (USE) questionnaire, after completing the design tasks in Method $-B \& C$. Both of the methods $B \& C$ are highly evaluated. The mean scores for Usefulness, Ease of use, Ease of learning and Satisfaction are given in Table 5. Overall average scores for all the USE dimensions are 80.90 and 80.92 for Method $-B \& C$ respectively.
Table 5: The usability levels for each dimension in the USE Questionnaire (Usefulness, Satisfaction, and Ease of use).

\begin{tabular}{lcc}
$\begin{array}{l}\text { Usability } \\
\text { Dimension }\end{array}$ & $\begin{array}{c}\text { Score } \\
\text { (Method B) }\end{array}$ & $\begin{array}{c}\text { Score } \\
\text { (Method C) }\end{array}$ \\
\hline Usefulness & $75.83 \%$ & $76.50 \%$ \\
\hline Ease of use & $80.00 \%$ & $78.54 \%$ \\
\hline Ease of learning & $85.33 \%$ & $86.00 \%$ \\
\hline Satisfaction & $82.47 \%$ & $82.66 \%$
\end{tabular}

Historically the scaled USE score of $80 \%$ and above is considered an acceptable threshold for good evaluation.

After completing design tasks in Method - B \& $C$, participants also recorded their opinion on the effectiveness of spatial analytics and visualizations in real-life architecture and building designing. Table 2 shows the exact questions which were asked from the participants and their responses. The user responses recorded are all above average (i.e. 3.7 and above out of 5). In the opinion of participants, such analytic tools can be a valuable addition into traditional architecture designing. They can help architects and designers in making informed decisions at every phase during the design process. They also believe that to some extent the static and dynamic spatial measures are valid representative of how human move in space and also that such tools can be adopted into professional architecture design pipeline.

\section{CONCLUSION AND DISCUSSION}

We have presented a spatial analysis tool for designing humanaware environments. Different from other simulation tools representing similar design metrics, our tool is readily integrated into a professional architecture pipeline (e.g. Autodesk Revit ${ }^{\circledR}$ ) for demonstration and evaluation purposes. Beyond static spatial analyses, the tool enables dynamic crowd simulations to investigate the impact of an environment on the building occupants, all interactively and in real-time. Our study demonstrates that, using our tools, 
architects can progressively refine their design solutions to improve developed designs in terms of accessibility, visibility, organization, crowd flow and walking distances. Note that the tool itself, however, isn't bounded to any specific metric and more measures can be adopted. A users' questionnaire demonstrates the usability and effectiveness of the tool in supporting architects' decision-making. In this work we have considered a limited number of static and dynamic analyses. For the dynamic crowd-based measures, we only test one agents' distribution (i.e. 75 agents, uniformly distributed in sub-groups with diametric targets).Future work will involve running more advanced static analyses incorporating representative description of user activities. More advanced dynamic analyses will involve testing different distribution of agents. We will also consider more advanced scenarios where heterogeneous agents will be confronted with evacuation procedures while accounting for agent groups and psychological factors (e.g. stress and panic). Machine learning techniques will be evaluated to speed up the computations. Also, this work doesn't present a comparison between static (Design Method - B) and dynamic (Design Method - C) tools and this is left for the future work.

In the current study, we operated at very high level of abstraction where users could not see furniture and other decor items. Further studies will improve the level-of-detail to have more informed design iterations. In the future we will also consult with experts to evaluate the quality of the produced building layouts. Expert architects will also be introduced to the tool and asked to participate in the study.

Despite the rigid boundaries of this study, we argue that the proposed tool can be further extended to incorporate additional static and dynamic analyses. Such analyses, coupled with advanced data analytics and visualization as the ones proposed in this study, hold promise to mark a major change in the architectural design work-flow towards the design of environment that best support users' needs and behaviors.

\section{ACKNOWLEDGMENTS}

This research has been partially funded by grants from the NSERC Discovery and Create programs, and in part by NSF IIS-1703883, NSF S\&AS-1723869, DARPA SocialSim-W911NF-17-C-0098, and the Murray Fellowship.

\section{REFERENCES}

Sonit Bafna. 2003. Space Syntax: A Brief Introduction to Its Logic and Analytical Techniques. Environment and Behavior 35, 1 (2003), 17-29.

Lola Ben-Alon and Rafael Sacks. 2017. Simulating the behavior of trade crews in construction using agents and building information modeling. Automation in Construction 74 (2017), 12-27.

Glen Berseth, Muhammad Usman, Brandon Haworth, Mubbasir Kapadia, and Petros Faloutsos. 2015. Environment optimization for crowd evacuation. CAVW 26, 3-4 (2015), 377-386.

Mehul Bhatt, Carl Schultz, and Minqian Huang. 2012. The Shape of Empty Space: Human-Centred Cognitive Foundations in Computing for Spatial Design. In Visual Languages and Human-Centric Computing. 33-40.

Isaac Guedi Capeluto and Boris Plotnikov. 2017. A method for the generation of climate-based, context-dependent parametric solar envelopes. Architectural Science 60, 5 (2017), 395-407.

Vincius J Cassol, Estêvão Smania Testa, Cláudio Rosito Jung, Muhammad Usman, Petros Faloutsos, Glen Berseth, Mubbasir Kapadia, Norman I Badler, and Soraia Raupp Musse. 2017. Evaluating and Optimizing Evacuation Plans for Crowd Egress. CG \& A 37, 4 (2017), 60-71.
Aslı Cekmis, Ișıl Hacıhasanoğlu, and Michael J Ostwald. 2014. A computational model for accommodating spatial uncertainty: Predicting inhabitation patterns in openplanned spaces. Building and Environment 73 (2014), 115-126.

Nilay Chakraborty, Glen Berseth, Brandon Haworth, Petros Faloutsos, Muhammad Usman, and Mubbasir Kapadia. 2017. Crowd Sourced Co-design of Floor Plans Using Simulation Guided Games. In Motion in Games (MIG '17). ACM, New York, NY, USA, Article 1, 5 pages. https://doi.org/10.1145/3136457.3136463

Qingyan Yan Chen. 2004. Using computational tools to factor wind into architectural environment design. Energy and buildings 36, 12 (2004), 1197-1209.

Mei Ling Chu, Paolo Parigi, Kincho Law, and Jean-Claude Latombe. 2014. Modeling social behaviors in an evacuation simulator. CAVW 25, 3-4 (2014), 373-382.

Joseph Clarke. 2007. Energy simulation in building design. Routledge.

Ren-Jye Dzeng, Wei-Chih Wang, Fan-Yi Hsiao, and Yan-Qing Xie. 2015. An ActivityBased Simulation Model for Assessing Function Space Assignment for Buildings: A Service Performance Perspective. Computer-Aided Civil and Infrastructure Engineering 30, 12 (2015), 935-950.

Anders Ekholm. 2001. Modelling of user activities in building design. In eCAADe. Helsinki, 67-72.

Linton C Freeman. 1978. Centrality in social networks conceptual clarification. Social networks 1, 3 (1978), 215-239.

John J Fruin. 1971. Pedestrian planning and design. Metropolitan Association of Urban Designers and Environmental Planners.

Jorge Gil, Eime Tobari, Maia Lemlij, Anna Rose, and AR Penn. 2009. The differentiating behaviour of shoppers: clustering of individual movement traces in a supermarket. (2009).

Rhys Goldstein, Alex Tessier, and Azam Khan. 2010. Schedule-calibrated occupant behavior simulation. In Spring Simulation Multiconference. Society for Computer Simulation International, 180.

Brandon Haworth, Muhammad Usman, Glen Berseth, Mubbasir Kapadia, and Petros Faloutsos. 2017a. On density-flow relationships during crowd evacuation. $C A V W$ 28, 3-4 (2017).

Brandon Haworth, Muhammad Usman, Glen Berseth, Mahyar Khayatkhoei, Mubbasir Kapadia, and Petros Faloutsos. 2017b. CODE: Crowd-optimized design of environments. CAVW (2017)

Nicolas Heess, Srinivasan Sriram, Jay Lemmon, Josh Merel, Greg Wayne, Yuval Tassa, Tom Erez, Ziyu Wang, Ali Eslami, Martin Riedmiller, et al. 2017. Emergence of locomotion behaviours in rich environments. arXiv preprint arXiv:1707.02286 (2017).

Dirk Helbing and Peter Molnar. 1995. Social force model for pedestrian dynamics. Physical review E 51, 5 (1995), 4282.

Bill Hillier and Julienne Hanson. 1984. The social logic of space, 1984. Press syndicate of the University of Cambridge (1984).

Christoph Hölscher and Martin Brösamle. 2007. Capturing indoor wayfinding strategies and differences in spatial knowledge with space syntax. In Space Syntax Symposium.

Christoph Hölscher, Simon J Büchner, Tobias Meilinger, and Gerhard Strube. 2009. Adaptivity of wayfinding strategies in a multi-building ensemble: The effects of spatial structure, task requirements, and metric information. Environmental Psychology 29, 2 (2009), 208-219.

Christoph Hölscher, Tobias Meilinger, Georg Vrachliotis, Martin Brösamle, and Markus Knauff. 2005. Finding the Way Inside: Linking Architectural Design Analysis and Cognitive Processes. In Spatial Cognition: Reasoning, Action, Interaction. Springer, $1-23$.

Seung Wan Hong and Yun Gil Lee. 2018. The Effects of Human Behavior Simulation on Architecture Major Students' Fire Egress Planning. Asian Architecture and Building Engineering 17, 1 (2018), 125-132.

Seung Wan Hong, Davide Schaumann, and Yehuda E. Kalay. 2016. Human behavior simulation in architectural design projects: An observational study in an academic course. Computers, Environment and Urban Systems 60 (2016), 1-11.

Yehuda E. Kalay. 2004. Architecture's new media: Principles, theories, and methods of computer-aided design. MIT Press, Cambridge.

Mubbasir Kapadia, Nuria Pelechano, Jan Allbeck, and Norm Badler. 2015. Virtual Crowds: Steps Toward Behavioral Realism. Synthesis Lectures on Visual Computing 7, 4 (2015), 1-270.

Mubbasir Kapadia, Alexander Shoulson, Cyril Steimer, Samuel Oberholzer, Robert W. Sumner, and Markus Gross. 2016. An event-centric approach to authoring stories in crowds. In MIG. ACM, 15-24.

Mubbasir Kapadia, Shawn Singh, William Hewlett, and Petros Faloutsos. 2009. Egocentric affordance fields in pedestrian steering. In I3D, Vol. 1. ACM, New York, NY, USA, 215.

Ioannis Karamouzas, Peter Heil, Pascal Van Beek, and Mark H. Overmars. 2009. A predictive collision avoidance model for pedestrian simulation. In Lecture Notes in Computer Science, Vol. 5884. Springer, 41-52.

Tae Wan Kim and Martin Fischer. 2014a. Automated generation of user activity-space pairs in space-use analysis. Construction Engineering and Management 140, 5 (2014), 04014007.

Tae Wan Kim and Martin Fischer. 2014b. Ontology for representing building users, activities in space-use analysis. Construction Engineering and Management 140, 8 (2014), 04014035.

Brian Lawson. 2004. What Designers Know. Architectural Press. 
Arnold M Lund. 2001. Measuring usability with the use questionnaire12. Usability interface 8, 2 (2001), 3-6.

Mary Lou Maher, Simeon J. Simoff, and John Mitchell. 1997. Formalising building requirements using an Activity/Space Model. Automation in Construction 6, 2 (1997), 77-95.

Xiaoshan Pan, Charles S. Han, Ken Dauber, and Kincho H. Law. 2007. A multi-agent based framework for the simulation of human and social behaviors during emergency evacuations. Ai \& Society 22, 2 (2007), 113-132.

Nuria Pelechano, Jan M Allbeck, Mubbasir Kapadia, and Norman I Badler. 2016. Simulating Heterogeneous Crowds with Interactive Behaviors. CRC Press.

Xue Bin Peng, Glen Berseth, KangKang Yin, and Michiel Van De Panne. 2017. Deeploco: Dynamic locomotion skills using hierarchical deep reinforcement learning. TOG 36, 4 (2017), 41.

Craig W Reynolds. 1987. Flocks, herds and schools: A distributed behavioral model SIGGRAPH 21, 4, 25-34.

Horst Rittel. 1971. Some principles for the design of an educational system for design Architectural Education 26, 1-2 (1971), 16-27.

Siobhan Rockcastle and Marilyne Andersen. 2014. Measuring the dynamics of contrast \& daylight variability in architecture: A proof-of-concept methodology. Building and Environment 81 (2014), 320-333.

Kerstin Sailer, Andrew Budgen, Nathan Lonsdale, Alasdair Turner, and Alan Penn. 2009 Comparative studies of offices pre and post, how changing spatial configurations affect organisational behaviours. (2009)

Davide Schaumann, Simon Breslav, Rhys Goldstein, Azam Khan, and Yehuda E. Kalay 2017a. Simulating use scenarios in hospitals using multi-agent narratives. BPS 10 , 5-6 (2017), 636-652.

Davide Schaumann, Kartikeya Date, and Yehuda E. Kalay. 2017b. An Event Modeling Language (EML) to Simulate Use Patterns in Built Environments. In Simulation for Architecture \& Urban Design. Toronto, 189-196.

Carl Schultz and Mehul Bhatt. 2012. Multimodal spatial data access for architecture design assistance. AI EDAM 26, Special Issue 02 (2012), 177-203.

Weilin Shen, Xiaoling Zhang, Geoffrey Qiping Shen, and Terrence Fernando. 2013 The User Pre-Occupancy Evaluation Method in designer, client communication in early design stage: A case study. Automation in Construction 32, Supplement C
(2013), 112-124.

Herbert A. Simon. 1969. The Sciences of the Artificial (mit press ed.). Cambridge, MA. Shawn Singh, Mubbasir Kapadia, Petros Faloutsos, and Glenn Reinman. 2009. An Open Framework for Developing, Evaluating, and Sharing Steering Algorithms. In MIG. Springer, 158-169.

Shawn Singh, Mubbasir Kapadia, Billy Hewlett, Glenn Reinman, and Petros Faloutsos. 2011. A modular framework for adaptive agent-based steering. In I3D. ACM, 9 .

Arthur E Stamps III. 2002. Entropy, visual diversity, and preference. General psychology 129, 3 (2002), 300-320.

Hosna Tashakkori, Abbas Rajabifard, and Mohsen Kalantari. 2015. A new 3D indoor/outdoor spatial model for indoor emergency response facilitation. Building and Environment 89 (2015), 170-182.

Alasdair Turner. 2001. A program to perform visibility graph analysis. In Space Syntax Symposium. 31-1.

Alasdair Turner, Maria Doxa, David O'sullivan, and Alan Penn. 2001. From isovists to visibility graphs: a methodology for the analysis of architectural space. Environment and Planning B: Planning and design 28, 1 (2001), 103-121.

Muhammad Usman, Brandon Haworth, Glen Berseth, Mubbasir Kapadia, and Petros Faloutsos. 2017. Perceptual Evaluation of Space in Virtual Environments. In MIG. ACM, Article 16, 10 pages.

Jur Van Den Berg, Stephen J. Guy, Ming Lin, and Dinesh Manocha. 2011. Reciprocal n-body collision avoidance. In Advanced Robotics. Vol. 70. 3-19.

Michael Weizmann, Oded Amir, and Yasha Jacob Grobman. 2017. Topological interlocking in architecture: A new design method and computational tool for designing building floors. Architectural Computing 15, 2 (2017), 107-118.

Leonard Wohl, Roland Gorzkowski, Nicole Fox, and Mubbasir Kapadia. 2016. KINDLING: A Game Platform for Crowd-sourcing Fire Evacuation Data. In Motion in Games (MIG '16). ACM, New York, NY, USA, 205-205. https://doi.org/10.1145/ 2994258.2994289

Wei Yan and Yehuda E. Kalay. 2004. Simulating the behavior of users in built environments. Architectural and Planning (2004), 371-384.

John Zeisel. 1984. Inquiry by design: Tools for environment-behaviour research. Number 5 . CUP archive. 